www. revistad yo.com

\title{
Estudio de la aplicabilidad de lean management en la industria minera
}

\author{
Jordi Fortuny-Santos, Patxi Ruiz-de-Arbulo López, Itziar Luján-Blanco \\ Recibido: 01 de Enero de 2021 \\ https://doi.org/10.37610/dyo.v0i73.595 \\ Aceptado: 15 de Enero de 2021
}

\section{Resumen}

Este trabajo explora, por medio de una revisión sistemática de la literatura, la aplicabilidad de la gestión lean en las empresas mineras. Se observa que la adopción se realiza por la existencia de presiones y como factores determinantes se tienen en cuenta la presencia de agentes facilitadores, la existencia de barreras y los líderes. Se concluye que en grandes empresas de todo el mundo, la implantación previa de otras herramientas de gestión facilita la implantación de las herramientas lean, y que a pesar de las diferencias con otros sectores, es posible mejorar la eficiencia de la industria minera a través de la gestión lean.

\section{Palabras clave}

lean, minería, JIT, facilitadores, mejora

\section{Introducción}

Cuando se redactan estas líneas se cumplen 30 años de la publicación, en 1990, de la versión original en inglés del libro "La máquina que cambió el mundo" (Womack et al., 1990). Dicho trabajo contribuyó enormemente a la difusión de las conclusiones del proyecto de investigación International Motor Vehicle Program (IMVP) del Instituto Tecnológico de Massachusetts (MIT) donde se originó el término "lean" (esbelto) para referirse el aspecto de algunas plantas de producción de automóviles que eran capaces de operar eficientemente con menor volumen de recursos que las convencionales. Por extensión, el término se aplicó

Jordi Fortuny-Santos*
jordi.fortuny@upc.edu
ID ORCID: 0000-0001-8106-3385
Patxi Ruiz-de-Arbulo López **
patxi.ruizdearbulo@ehu.eus
ID ORCID: 0000-0003-4881-0304
Itziar Luján-Blanco ***
itziar.lujan@upc.edu
ORCID: 0000-0002-2869-4708
* Departamento de Organización de Empresas, Universitat
Politècnica de Catalunya, Manresa, España.
Grupo OPE-PROTHIUS 2017 SGR 314 .
** Departamento de Organización de Empresas, Universidad
del País Vasco /Euskal Herriko Unibertsitatea, Bilbao, España
*** Departamento de Organización de Empresas, Universitat
Politècnica de Catalunya, Manresa, España

inmediatamente al sistema de gestión empleado en dichas plantas como "lean production". Womack et al. (1990) presentan a Toyota como el principal exponente de gestión lean; enraizando la producción lean en el sistema desarrollado por Toyota después de la Segunda Guerra Mundial, denominado simplemente Toyota production system (TPS) (Ohno, 1988) y conocido en Occidente como just-in-time (Sugimori et al, 1977).

Womack et al. (1990) y los resultados del estudio IMVP muestran la gestión lean como paradigma de la eficiencia operacional y no hay duda que su sistema de producción ha contribuido a convertir a Toyota en una de las principales compañía automovilísticas del mundo (Dunstan et al., 2006).

El objetivo fundamental de la producción lean es la creación de valor para el cliente Womack et al. (1990). Por dicho motivo, se orienta a eliminar todas aquellas actividades que no añaden valor -pero sí costes- en la fabricación del producto (a las que denominamos muda en japonés), mediante un proceso de mejora continua en el cual la implicación del personal es fundamental (Ohno, 1988, Sugumori et al. 1977). Puesto que no existe una definición formal de producción lean o de gestión lean (Pettersen, 2009), podemos considerar como tal a aquella que aplica los principios anteriores de eliminación de muda y mejora continua participativa. Para lograr sus propósitos, las empresas lean emplean distintas prácticas (Dieste et al, 2019), imitando a Toyota, aunque tampoco puede determinarse taxativamente qué técnicas o herramientas debe aplicar una empresa para considerarse lean (Shah y Ward, 2007). 
Primero Ohno (1988) y después Womack et al. (1990) afirmaron que los principios de TPS / LM se pueden aplicar a cualquier sector. Ohno (1988) insiste en la necesidad de concentrarse en los principios, no en las herramientas en concreto -puesto que los occidentales que empezaban a conocer el sistema JIT parecían sólo interesarse por el uso de tarjetas kanban-. Desde entonces, queda abierto el debate sobre la universalidad de LM. Puesto que las características de las distintas actividades productivas difieren de las del sector automovilístico donde nació la gestión lean, es de gran importancia conocer cómo se puede aplicar LM en cada sector: qué circunstancias lo favorecen; qué elementos actúan en contra; qué prácticas son aplicables; qué nivel de desarrollo alcanza la implantación; qué resultados se obtienen.

En los últimos años, se han publicado multitud de trabajos sobre la aplicación de LM a sectores bien distintos del sector de automóvil, como pueden ser el sector sanitario o el de la construcción (Para una revisión más amplia se recomienda consultar el trabajo de Netland y Powell, 2017) pero sigue siendo necesario el estudio de los determinantes de la implantación y la caracterización de LM en cada uno de los sectores donde se aplique. En concreto, en este trabajo, nos planteamos la aplicabilidad de LM en el sector minero, en sentido amplio, incluyendo tanto las explotaciones subterráneas como las explotaciones a cielo abierto, las canteras y la extracción de petróleo y gas, teniendo en cuenta que el sector así definido es heterogéneo por lo que se refiere a productos y métodos de explotación, además de las disparidades entre empresas que pueda presentar cualquier sector (por ejemplo, la dimensión de las empresas). El interés del estudio del sector minero se basa en la importancia económica del mismo: la industria minera suministra las materias primas que son la base de la producción de multitud de productos de uso cotidiano y proporciona metales, minerales, rocas y fuentes de energía que son fundamentales para nuestro bienestar. Por ejemplo, el uso de los teléfonos móviles no sería posible si en sus circuitos no incluyeran componentes electrónicos de elevadas prestaciones como los condensadores de tántalo. Dicho metal se obtiene del coltán (roca que contiene los minerales columbita y tantalita), que se ha popularizado en los medios de comunicación en los últimos años por proceder, en parte, de regiones de África con elevada inestabilidad (El Periódico, 2020). Otro ejemplo clave para el futuro inmediato es la obtención del cobalto, imprescindible para fabricar baterías de alto rendimiento para equipos electrónicos (ordenadores, teléfonos) y para vehículos eléctricos. Sin embargo, la mayor mina de cobalto del mundo tuvo que cerrar por falta de rentabilidad (Robertson, 2019). Frente a estas circunstancias, la Unión Europea reconoce la importancia estratégica del sector minero y la necesidad de asegurar el suministro de materias primas esenciales a la industria de la Unión, evitando así depender de terceros países (Europa, 2009).
Para garantizar su futuro, las empresas mineras, al igual que las de cualquier otro sector, deben elaborar una estrategia que afiance su viabilidad desde distintas perspectivas -entre ellas, la correspondiente al área de operaciones-. Puesto que la explotación minera no deja de ser un tipo de industria, seguro que puede beneficiarse de distintas técnicas de organización industrial (Hattingh y Keys, 2010). De hecho, Claassen (2016) comprueba, en 22 minas en Sudáfrica, que cada mina emplea una media de cinco técnicas de gestión procedentes de la industria manufacturera. Siguiendo la estela de Toyota, multitud de empresas de sectores diversos, incluyendo el minero, han recurrido a la implantación de técnicas y herramientas de LM para mejorar su eficiencia operacional, su calidad y su capacidad de respuesta (Iranmanesh et al., 2019), ante lo cual volvemos a insistir en la necesidad de caracterizar la implantación de LM en cada sector. Por ejemplo, el sector minero está plagado de procesos sin valor añadido (Flynn y Vlok, 2015) que comportan falta de productividad, ineficiencias, contaminación, abuso de los recursos y falta de calidad, mientras que LM se ha revelado como un arma potente en la identificación y eliminación de despilfarros de recursos. No obstante, a nadie escapa que hay notables diferencias entre el trabajo minero y el trabajo en una fábrica de automóviles (Chlebus et al, 2015). Omitimos aquí mencionarlas pues se encuentran tabuladas en Dunstan et al. (2006) y posteriormente se detallan en Helman (2012). No obstante, a partir de este último trabajo, mencionamos algunas diferencias que parecen ir claramente en contra de los requisitos de la producción lean:

La explotación minera es prácticamente un proceso continuo en sistema push, que va alimentando a refinerías, fundiciones o plantas de enriquecimiento. Algunas de estas instalaciones funcionan en régimen de producción continua, por lo que no se puede detener la producción como haría una fábrica de producción discreta como una fábrica de automóviles. Mientras que la producción industrial ofrece un entorno más o menos estable, la actividad minera viene condicionada por la geología del terreno y ésta ofrece múltiples variaciones e incertezas. La producción industrial se realiza en una fábrica de una determinada dimensión, mientras que la explotación minera puede estar dispersa en un área de centenares de kilómetros cuadrados. Ante estas diferencias, se hace imprescindible explorar la aplicabilidad de LM en el sector minero, sus características y determinantes, con especial énfasis en la dinámica del cambio que supone en la empresa minera la implantación de LM.

\section{Metodología del estudio}

El método de investigación empleado en este trabajo es una revisión sistemática de la literatura con la que pretendemos determinar las características y determinantes de LM en el sector minero a través de las publicaciones existentes sobre aplicación de LM en empresas mineras. La revisión sistemática de la literatura es una herramienta que 
se ha empleado repetidamente en el campo de la gestión de operaciones (Tavares-Thomé et al., 2016; Fortuny-Santos et al., 2020) e incluso para analizar la aplicación de la gestión lean en sectores concretos como el sanitario (Brandao de Souza, 2009). La metodología seguida para realizar la búsqueda sistemática es similar a la descrita por Dieste et al. (2019), pero además de un análisis de contenidos hemos realizado un estudio bibliométrico (Boar et al., 2020).

Para realizar la revisión de la literatura, se consideró el uso de dos grandes fuentes de referencia: Web of Science (WOS) y Scopus (Thunnissen y Gallardo-Gallardo, 2019). Los resultados que se obtienen con cada una de ellas no son coincidentes pero ciertamente entre las dos existe un fuerte solapamiento. Puesto que también los formatos de los datos que se recuperan son distintos, pensando en realizar un estudio bibliométrico con la herramienta VOSViewer, se optó por emplear una única base de datos y se escogió Scopus por ser más amplia que WOS. La metodología escogida para esta investigación tiene la debilidad de recuperar aparentemente sólo aquellos proyectos empresariales que han despertado el interés de algún investigador pero, como se pudo constatar en los resultados obtenidos, Scopus incluye, junto a las publicaciones más académicas, otras revistas orientadas a los profesionales del sector, donde son las propias empresas quienes comunican sus logros.

Antes de iniciar la búsqueda sistemática, se organizaron sesiones de brainstorming para decidir los términos de búsqueda (en los campos título, resumen o palabras clave de los documentos). Por un lado se requerían palabras que hicieran referencia al mundo minero y por otro a la gestión lean. Se optó por especificar términos en inglés lo que nos aboca a recuperar sólo los trabajos redactados en dicho idioma -que, según Thunnissen y Gallardo-Gallardo (2019), por su capacidad de difusión a nivel internacional, acaban siendolos más relevantes- o aquellos que, redactados en otra lengua, cuenten con palabras clave o resumen en inglés, siendo ésta una práctica muy habitual en la literatura científica. Debido al interés que tendría para nosotros hallar trabajos basados en empresas españolas, se realizó una ulterior búsqueda con términos traducidos al castellano, pero no se logró recuperar ningún documento adicional. Finalmente, en las cadenas de búsqueda se incluyeron las combinaciones de dos categorías de palabras clave. Por un lado, los términos mining, minerals, quarry, ore, oil y gas para abarcar los distintos subsectores dentro de la minería. Por otro lado, se incluyeron lean, Toyota production system, JIT o Just in time y las principales herramientas de LM: Total Productive Maintenance, TPM, kanban, kaizen, VSM, value stream map o 5S. Se observó que la estrategia de búsqueda así formulada era más completa que la definida por Lööw (2019) en su revisión. Se excluyeron de la búsqueda los trabajos que contuvieran los términos data mining y text mining para evitar estudios relacionados con la llamada "minería de datos". Esta metodología tiene la debilidad que sólo se podrán analizar aquellos documentos que hayan sido localizados por las cadenas de búsqueda, pudiendo quedar trabajos importantes fuera de nuestro estudio. Es por ello que se procuró que la lista de términos fuese exhaustiva.

No se establecieron otros límites de búsqueda, ni por nacionalidad, ni por tipo de documento ni por antigüedad. Puesto que la búsqueda bibliográfica se realizó entre enero y febrero de 2020, se recuperaron todos los trabajos con fecha de publicación hasta diciembre de 2019. Se localizaron 164 referencias y, tras una lectura del título y del resumen para comprobar que los documentos se ajustaban a nuestros propósitos, se seleccionaron 68 trabajos. Estos documentos se emplearon para el análisis bibliométrico, para el cual se emplearon los programas informáticos Microsoft Excel y VOSviewer versión 1.6.14 disponible en www.vosviewer.com, pues es software de acceso libre creado en la Universidad de Leiden (Países Bajos). Posteriormente, durante la lectura de la selección de trabajos para analizar su contenido, se recopilaron algunos textos más que aparecían citados repetidamente, empleando la técnica denominada "bola de nieve" (Sayers, 2007).

Al realizar la búsqueda bibliográfica, se observó la existencia de tres trabajos de revisión previos (Nadeau et al., 2015; Seifullina et al., 2018; Lööw, 2019) aunque existen diferencias entre todos ellos por período cubierto, estrategia de búsqueda y objetivos del trabajo (por ejemplo, el trabajo de Nadeau et al. se orienta hacia la prevención de riesgos laborales).

En nuestra investigación, basándonos principalmente en los trabajos de naturaleza empírica (aunque también se han tenido en cuenta aquellos que aportan una discusión académica), hemos recogido, además de los datos bibliográficos de cada documento, los subsectores donde se ha implantado LM, las herramientas lean empleadas, la dimensión de las empresas (tomado nota, cuando es posible, del número de empleados o de la cifra de ventas) y los países donde se ha realizado la implantación. Para alcanzar los objetivos del estudio, hemos prestado especial atención a la dinámica del cambio que supone en la empresa la implantación de LM. Para ello se ha seguido el modelo de Martínez Ramos (2004) que incluye el análisis de las presiones internas o externas que afectaron la decisión de adoptar la filosofía lean, los factores que intervinieron en el proceso de implantación y las consecuencias o resultados de la implantación de LM y de su uso. Dicho modelo surge para analizar la implantación de sistemas contables (Ruiz de Arbulo et al., 2013) a partir de la integración de modelos propuestos por renombrados investigadores pero por su enfoque permite estudiar la introducción de otros tipos de novedades, explicando el proceso desde la perspectiva de sus antecedentes y sus consecuencias puesto que LM viene 
a sustituir a prácticas bien arraigadas así que la presencia de estímulos es fundamental. Por lo que se refiere a las presiones del entorno, los aspectos que influyen en el cambio de modo general se denominan motivadores, mientras que los agentes del cambio o catalizadores están asociados directamente con el cambio. Por lo que respecta a los factores que favorecen o dificultan la adopción, el modelo distingue entre favorecedores internos o enablers (elementos de carácter técnico organizacional), barreras (que dificultan o impiden el cambio) y líderes (papel de un líder como impulsor del proyecto).

\section{Análisis bibliométrico}

Aunque los documentos más antiguos que se recuperaron se publicaron en 1989, es a partir de 2006 cuando se observa un incremento sostenido en el número de trabajos, como muestra la Figura 1. Entre 1990 y 1992 no aparece ningún trabajo y entre 1993 y 2015 sólo se publican nueve. El ritmo de aparición de publicaciones aumenta especialmente desde 2015, año en que aparecieron siete trabajos. En 2019, último año analizado, fueron cinco. El número de citas ha aumentado rápidamente, mostrando el interés por este campo. Los trabajos con mayor impacto, por número de citas, corresponden a Dunstan et al. (2006) con 26 citas, Indrawati et al. (2015) con 25 citas, Chlebus et al. (2015) y Mishra et al. (2013) con 17 citas cada uno. No obstante, el $72 \%$ de los documentos revisados ha recibido menos de cinco citas. Cabe destacar además que un $37 \%$ de los títulos revisados corresponden a trabajos presentados en congresos, lo cual es habitual en un área emergente que no ha dado lugar todavía a muchas publicaciones en revistas.

Figura 1 Publicaciones y citas por año (desde 2006 hasta 2019).

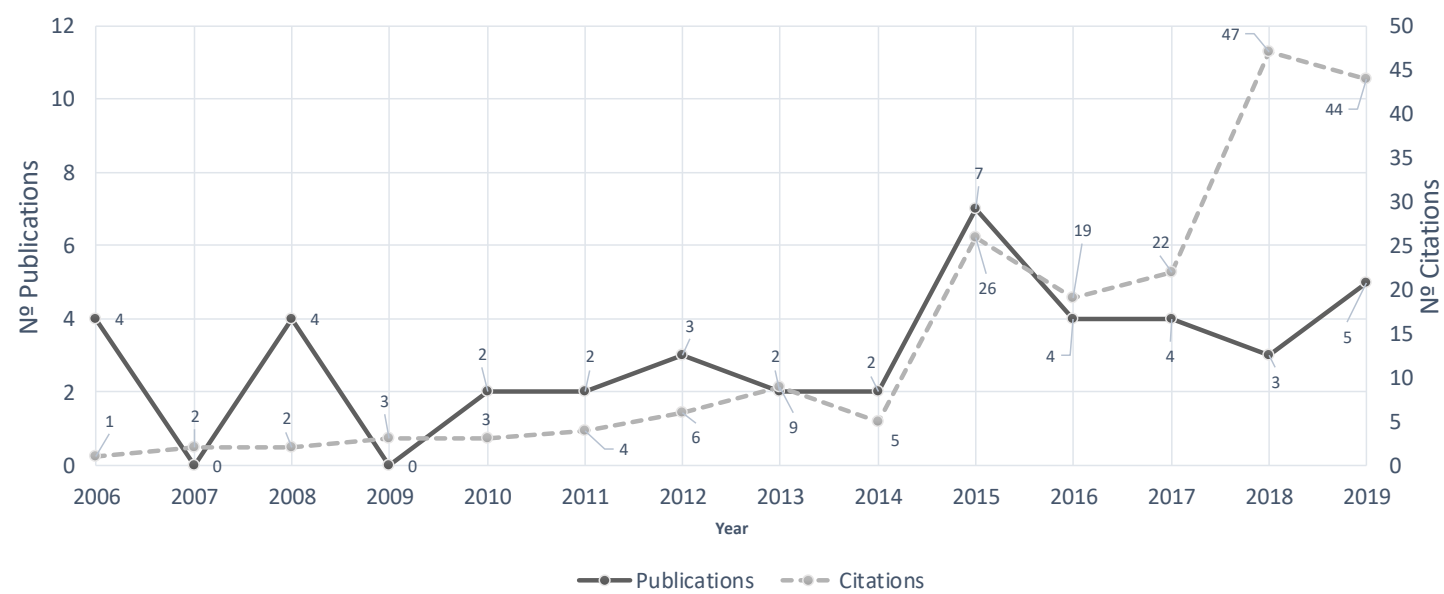

Los estudios relacionados con la aplicación de LM en la industria minera no aparecen en las principales revistas sobre gestión de operaciones. Según la clasificación realizada por Scopus, los trabajos sobre la aplicación de LM en la minería corresponden fundamentalmente a las ramas de ingeniería y de ciencias de la tierra (Figura 2), es decir, las áreas relacionadas con la actividad minera. El área de gestión aparece en tercer lugar pero a gran distancia. 
Figura 2 Ramas, según la clasificación de Scopus (en inglés), que han publicado trabajos sobre lean management en industrias mineras.

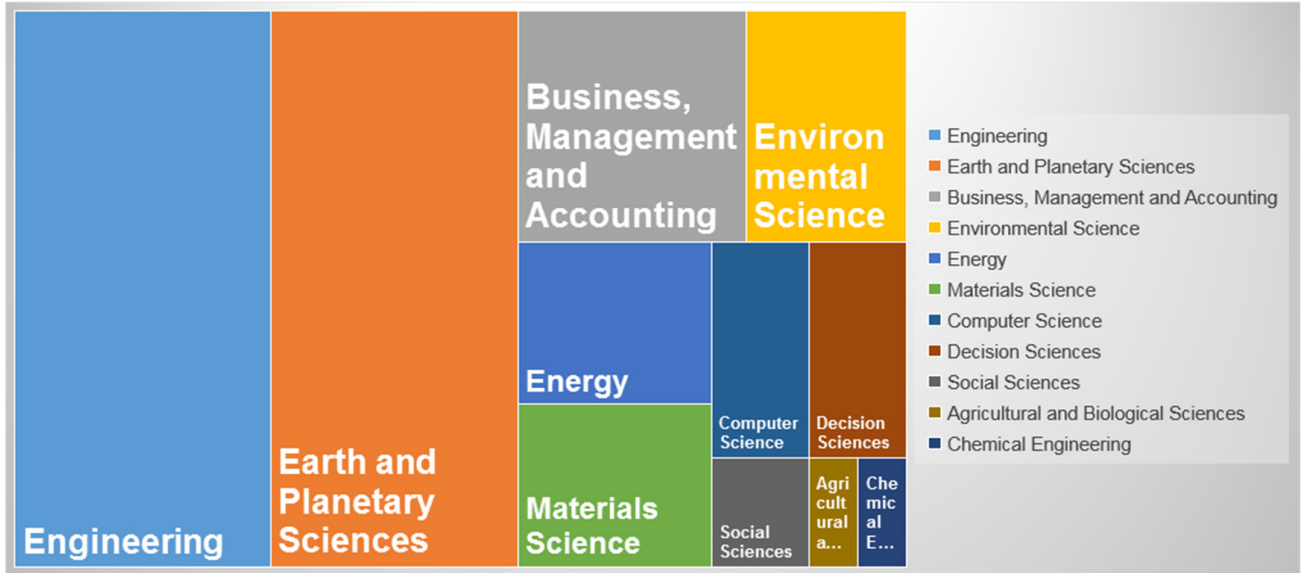

relaciona la producción lean en el ámbito minero con la mejora continua. Por otro, se identifica el término lean mining con aplicación de la producción lean y la gestión de la industria minera. Finalmente, aparece el término justin-time asociado a la automatización y a la ingeniería de procesos, es decir, haciendo referencia a la modernización de la mina.

Figura 3 de coocurrencias en el campo de LM aplicado a la industria minera.

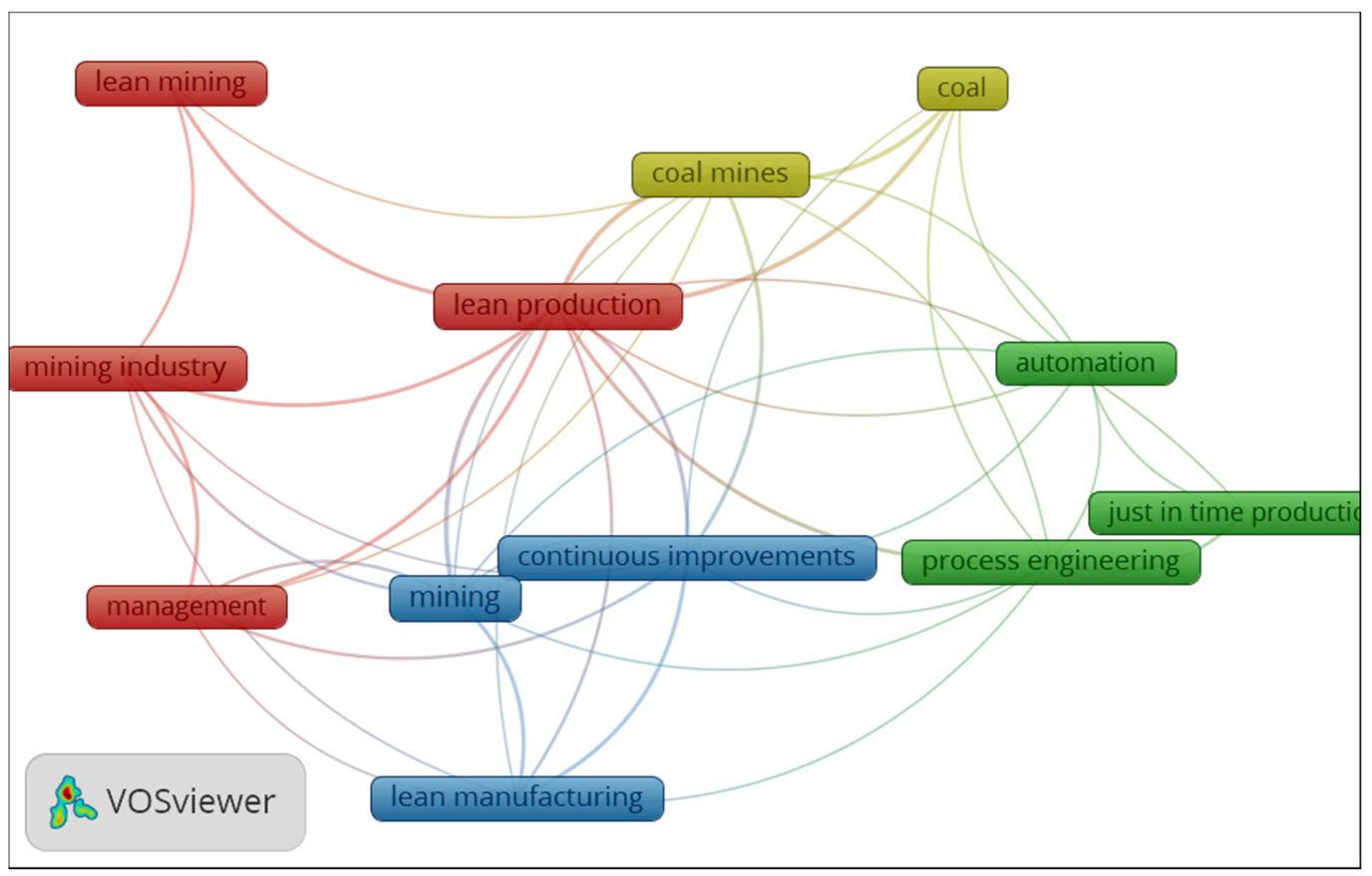




\section{Lean mining: resultados y discusión}

En 1989, el sector minero empieza a descubrir las entregas JIT (Strauss, 1989) como exigencia de sus clientes (como también suele suceder en el sector industrial) y otro trabajo (Anónimo, 1989) menciona la aplicación de un sistema de gestión de existencias JIT en una mina de carbón norteamericana. Por lo tanto, vemos que el sistema JIT llega al sector minero relativamente pronto. Cavender (1993) propone la aplicación del World Class Manufacturing (definido por él mismo como la combinación entre la gestión de existencias JIT y la calidad total) a la minería y menciona que dicha metodología -que viene a ser un sinónimo de la gestión lean- ya está implantada en grandes empresas como Rio Tinto. Volvemos a encontrar referencias al JIT en Simmons (1996), Valent y Haywood (2002) o Musingwini et al. (2003) que muestran como el acrónimo JIT se sigue empleando aun cuando ya se ha popularizado el término lean. Aunque lean y JIT se usen muchas veces como sinónimos, debemos recordar que no lo son, pues JIT es sólo una parte del sistema de producción de Toyota y precisamente en estos primeros trabajos notamos que los autores que emplean la denominación JIT se centran fundamentalmente en la gestión de existencias.

Chadwick (1995) utiliza por primera vez el concepto lean mining y éste se repite posteriormente en Vanegas (1999) como la manera de minimizar el tiempo de throughput, las existencias, los despilfarros de recursos y los retrabajos, es decir, objetivos típicos de la gestión lean. De manera similar a lo sucedido con lean healthcare, se ha generalizado el término lean mining para describir la aplicación de los principios lean al sector minero y ya cuenta con página web en el portal de la asociación Lean Global Network, creada por Womack, e incluso existe el Instituto Lean Mining (Mottola, 2018). Estas entidades contribuyen a difundir las buenas prácticas y potenciar la aplicación de LM en el sector minero.

Azzamouri et al. (2016) presentan lean mining como una manera de explotar mejor los recursos naturales con un menor consumo de factores productivos. Debido a la tradicional mala fama de las actividades mineras por su impacto ambiental, un sistema de gestión para la industria minera no puede dejar de lado el compromiso con la sostenibilidad. Por dicho motivo, Azzamouri et al. (2016) presentan el lean mining responsable enfocado a la explotación racional, sostenible a largo plazo.

Liu (2013) pone grandes esperanzas en la aplicación de LM en una mina de carbón: eliminar despilfarros de recursos para poder reducir costes; favorecer la flexibilidad para poder ajustar la producción a la demanda del mercado; mejorar la productividad laboral; mejorar el entorno de trabajo; reducir el coste de las existencias. Sin embargo, Castillo et al. (2014) constatan que, para las empresas mineras, LM sigue siendo una filosofía emergente para unos procesos y modelos de negocio que no se han modernizado. Según estos autores, LM promete ventajas competitivas y ahorros pero continúan insistiendo en la existencia de importantes limitaciones prácticas. No obstante, como fruto de su investigación recomiendan a las empresas que valoren la adopción de metodologías lean, especialmente considerando los retos a los que el sector se enfrenta. En su siguiente trabajo, Castillo et al. (2015) explican que hay pocas evidencias de la aceptación de LM en la minería y sólo unas pocas empresas se han embarcado en la transformación hacia la filosofía lean. Señalan igualmente la falta de investigación sobre la capacidad que tiene la metodología lean de mejorar la producción y el funcionamiento de la empresa. No obstante, hasta ese momento ya habían aparecido diversos trabajos de carácter empírico, entre los que destacaríamos: Yingling et al. (2000), Dunstan et al. (2006), Groeneveld (2006), Klippel et al. (2008a y 2008b), Roelandt (2008), Hattingh y Keys (2010), Haugen, (2013); muchos de los cuales aparecen citados en Castillo et al. (2014). A los anteriores, les seguirán trabajos como Flynn y Vlok (2015) Vanek et al. (2015) o Kęsek et al. (2019) pero a pesar de la existencia de todos estos trabajos, Makumbe et al. (2018) siguen afirmando que la implantación de LM en las empresas del sector minero es lenta y fragmentada.

Por países, se han aplicado (o se han hecho estudios para su aplicación) herramientas de LM en la industria minera en múltiples naciones donde el sector minero juega un papel importante: Alemania, Australia, Brasil, Bulgaria, Canadá, Chile, China, Eslovaquia, Estados Unidos, Ghana, Hungría, India, Indonesia, Irán, Marruecos, Noruega, Polonia, Reino Unido, República Checa, Rumanía, Rusia, Sudáfrica, Suecia, Tailandia o Zimbabue. Podemos ver que la lista abarca los cinco continentes y se extrañan algunos países que, a pesar de ser ricos en minerales, corresponden a zonas en conflicto o son naciones que suelen generar pocos trabajos científicos. Nos ha sorprendido no hallar en la literatura ninguna aplicación de LM en España, el país más rico en minerales de la Unión Europea. Esto abre la posibilidad de realizar un trabajo empírico en España para ver la situación real de las empresas. La diversidad de países prueba que LM no es algo exclusivo de las empresas japonesas o de la cultura japonesa, sino que se puede trasladar a otras culturas, aunque cada cultura pueda imprimirle sus variantes (Wong, 2007).

Por lo que se refiere a la naturaleza de las explotaciones donde se han aplicado herramientas de LM, encontramos una gran variedad de casos, algunos de ellos con evidencias en más de un país: arenas bituminosas, áridos, bauxita (mena de aluminio) e instalaciones de fundición de aluminio, amatista, carbón, cobre, crisotilo, diamantes, fluorita, fosfatos, hierro $\mathrm{y}$ las fundiciones de metales férreos y no férreos, oro, plata, petróleo y gas natural así como refinerías y plantas petroquímicas, platino, plomo o zinc. Además, los trabajos revisados cubren las fases de exploración, construcción, explotación (tanto subterránea como a cielo abierto), procesado posterior y mantenimiento. Por lo tanto, tenemos 
evidencias de que LM puede aplicarse a distintos subsectores y tipos de explotación, por lo que no podemos concluir que LM sea adecuado para ciertas actividades pero no para otras.

Por lo que se refiere a la dimensión de las empresas mineras, se trata siempre de empresas grandes. Por ejemplo, Vanek et al. (2015) describen la empresa checa OKD, única productora de carbón en el país, con más de 18000 empleados y una facturación de 1680 millones de dólares. En otros trabajos se citan empresas multinacionales como pueden ser Rio Tinto (Dunstan et al. 2006) -la más nombrada-, Anglo American (Hattingh and Keys, 2010) o Boliden (Haugen, 2013).

Las técnicas lean mencionadas en los trabajos empíricos o conceptuales son también múltiples. Así, se indica que se aplican o se sugiere que se podrían aplicar, entre otros: las 5 S (Baladrón y Alarcón, 2017), el kamishibai (Dunstan et al., 2006) cuadros heijunka, Value Stream Map (Kęsek et al., 2019), ir al sitio de trabajo para ver la fuente de los problemas (Lin y Ghodrati, 2011) y otros elementos de gestión visual (Flynn y Vlok, 2015); kaizen o mejora continua (Stecuła et al., 2017) e identificación de los distintos tipos de muda (muy interesante consultar la identificación recogida por Seifullina et al., 2018) así como uso de elementos de la calidad total (diagramas de Pareto, análisis de modos de fallo AMFE, control estadístico de procesos) y técnicas six sigma (Yuan et al., 2016); mantenimiento preventivo TPM (Stecuła et al., 2017; Brodny et al., 2017); elementos de gestión de recursos humanos y organización del trabajo como dar mayor responsabilidad y autonomía, crear equipos de trabajo (Sanda et al., 2011), estandarizar el trabajo, jidoka, trabajar en células, polivalencia; cambios rápidos; simulación; fijación de objetivos medibles; trabajo bajo pedido (JIT, pull), etc. No se observan diferencias entre estos términos y los empleados en las empresas industriales. E igual como sucede en el sector industrial, las empresas no emplean todas las herramientas ni aplican LM en todas las circunstancias. Hay empresas que sólo se han interesado por el mantenimiento; otras han empezado aplicando LM en la parte más industrial de su proceso (por ejemplo en un horno de fundición) y luego lo han extendido a la explotación minera.

Así como las técnicas de mejora de calidad six sigma se han unido al mundo lean cuando originalmente nada tenían que ver con el mismo (Spector, 2006), Haragovics y Mizsey (2014) identifican al análisis exergético como herramienta lean, en el sector del petróleo, pues es una herramienta fundamental para mejorar la eficiencia energética de los procesos.

Las presiones que han llevado a la implantación de herramientas de LM son múltiples, pero, tanto si se consideran motivadores genéricos como agentes del cambio, podría decirse que han llevado a la empresa a una pérdida de competitividad que amenaza su supervivencia. Por lo tanto, la implantación no es un divertimento sino una necesidad acuciante: la competencia mundial que ha llevado a la necesidad de producir a menor coste (Vanek et al.,
2015); la crisis financiera (Haragovics y Mizsey, 2014); las reconversiones sectoriales (Kęsek et al., 2019); la caída de precios de mercado que obliga a reducir costes (Dunstan et al., 2006); la variabilidad temporal de la demanda que obliga a ser flexible (Groeneveld, 2006); la necesidad de aumentar la producción para atender los pedidos; la necesidad de explotar reservas más profundas y de menor calidad, lo cual exige una mayor eficiencia (Mishra et al., 2013); la necesidad de tener la maquinaria disponible (Brodny et al., 2017); presiones sociales y legales en materia de prevención de riesgos laborales o de medioambiente (Yu et al., 2016); imitar la competencia (Dunstan et al., 2006); o simplemente el darse cuenta que el sector se queda anticuado, que requiere una mejora de su gestión (Castillo et al., 2014) que favorezca la innovación (Klippel et al., 2008b) y que manifiesta una baja productividad que acaba manifestándose con las quejas de los clientes por baja calidad o por retrasos en las entregas. Existe el caso particular descrito por Hattingh y Keys (2010) en el que se describe como el fabricante de equipo Sandvik trans4mine actúa como agente del cambio e impulsa a sus clientes, las empresas mineras, a adoptar LM.

Por lo que respecta a los factores que han facilitado la introducción de LM en la empresa, encontramos empresas que, antes de optar por LM, ya han implantado programas de mejora continua, Six sigma (Flynn y Vlok, 2015; Groeneveld, 2006), seguridad Dupont (Dunstan et al., 2006), formación del personal, gacetilla de la empresa, programas de fidelización del personal, etc. con lo que podríamos decir, por un lado, que se trata de empresas "inquietas" en cuanto a herramientas de gestión y por otro, que tanto los directivos como el resto del personal ya están acostumbrados a la introducción de nuevas técnicas, lo que evita que se sientan amenazados por la novedad. Esto es especialmente cierto para aquellas herramientas que exigen la implicación del personal (Flynn y Vlok, 2015). En este sentido, Sanda et al. (2011) reconocen que las mejoras provienen del conocimiento tácito del personal. Dentro de los facilitadores, cabe destacar también el soporte de consultores externos (Castillo et al., 2015) y el compromiso y liderazgo de la alta dirección (Castillo et al., 2014).

En los trabajos revisados, también aparecen algunas barreras que han dificultado la implantación: en primer lugar, la variabilidad intrínseca del trabajo causada por la naturaleza del terreno que dificulta la estandarización y las previsiones (por ejemplo, tras una voladura) es la causa más mencionada (Wijaya et al., 2009; Steinberg y De Tomi, 2010); resistencia al cambio y falta de fe en el proyecto; aspectos culturales y la costumbre de trabajar de una determinada manera (Castillo et al., 2014); elevada rotación del personal (Wade, 2008); sistemas de sugerencias inútiles Hattingh y Keys, 2010); estructuras funcionales herméticas, sin comunicación entre ellas (Flynn y Vlok, 2015); imposibilidad de confiar en el equipo mecánico. Como apuntan Dunstan et al. (2006), es normal que los trabajadores se sientan sorprendidos por las diferencias entre una empresa minera y una fábrica y esto suponga un elemento inicial de resistencia al cambio, pero 
aquí la dirección debe jugar un papel fundamental y dejar que el personal vaya solucionando problemas y tomando decisiones para darse cuenta que la metodología LM no está lejos de su trabajo. Sólo Helman (2012) detalla problemas de tipo técnico en la implantación de una herramienta en concreto, la tarjeta kanban, pues en la mina los materiales se mueven horizontalmente y verticalmente y se reúnen en cintas transportadoras.

Finalmente, para que una iniciativa alcance el éxito, es preciso que alguien (a veces denominado champion) asuma un papel de liderazgo, impulsando el proyecto, evitando que se estanque y reconduciéndolo cuando es necesario. En la mayoría de trabajos revisados no consta quien ejerce este papel. En otros se menciona la dirección (Boateng-Okrah y Appiah Fening, 2012), y específicamente una nueva dirección (Vanek et al. 2015) un consultor externo (Castillo et al, 2015; Dunstan et al., 2006), un grupo de expertos enviado por la central de la empresa (Roelandt, 2008), un equipo de investigadores (Azzamouri et al., 2016; Lin and Ghodrati, 2011) o los equipos de mejora (Flynn y Vlok, 2015). Hattingh y Keys (2010) recogen un caso en el que, si los consultores se van, las mejoras se detienen. Con esto se ve la importancia del liderazgo.

Las iniciativas han dado sus frutos y todos los trabajos empíricos mencionan sus resultados cualitativos o cuantitativos tras la aplicación de LM. Algunas son mejoras directamente relacionadas con las bondades habitualmente asociadas a LM; otras son mejoras sucedidas en otros campos de la gestión pero que derivan de la transformación sucedida en la empresa. Se mencionan mejora de la productividad, reducción de los tiempos de paro, reducción de costes, aumento en la capacidad y en las cantidades extraídas (por ejemplo, Klippel et al. (2008b) y Flynn y Vlok (2015) señalan que en una empresa brasileña el volumen extraído mensualmente creció un $43.6 \%$, pero al mismo tiempo el coste por tonelada disminuyó un 32.9\% ), mejora en la eficiencia energética, disponibilidad de la maquinaria, mejoras en la facturación, mejor retención del personal, mejoras en las condiciones de seguridad e higiene (por ejemplo, trabajar con menos polvo), mejora de la calidad y el medio ambiente, disponibilidad de información, reducción de existencias, creación de procedimientos de trabajo, reducción de costes gracias a las ideas del personal y de las empresas subcontratadas (Kęsek et al., 2019), mejora en general del proceso de gestión (Mikhalchenko y Rubanik, 2019). En resumen, Flynn y Vlok (2015), concluyen los resultados de la aplicación de LM en la industria minera se pueden considerar un éxito.

\section{Conclusiones}

De la revisión de la literatura se desprende que, desde 1989 han ido surgiendo trabajos de investigación sobre la aplicación de LM en la industria minera. Mientras en las primeras publicaciones se hace referencia a la gestión de existencias JIT, a mediados de la década de 1990 se empieza a emplear el término lean e incluso surge la expresión lean mining que se ha ido consolidando -más en la divulgación profesional que en la investigación académica-. El ritmo de aparición de trabajos en revistas y en congresos ha acelerado en los últimos cinco años, por lo que puede hablarse de una nueva línea de investigación, aunque todavía esté en su infancia y se concentre en dar a conocer las experiencias de las empresas. Estas circunstancias recuerdan el origen de otra línea de investigación surgida en la misma época, la que estudia las relaciones entre LM y sostenibilidad. En dicha línea, en los últimos años, se ha pasado del estudio de casos a otros tipos de metodologías empíricas que permiten analizar correlaciones entre variables.

Los estudios empíricos disponibles revelan que grandes empresas mineras, en todo tipo de explotaciones repartidas por los cinco continentes, adoptan herramientas de LM al igual que las empresas industriales. En la gran mayoría de casos, la adopción se origina cuando las empresas advierten que su competitividad se ve comprometida por circunstancias del entorno exterior (por ejemplo, caídas de precios de mercado). No constan presiones por parte de los clientes para adoptar el modelo LM, más allá de realizar entregas JIT. Se observa que muchas de estas empresas, previamente a la introducción de LM, ya han estado aplicando otras herramientas de gestión, lo cual contribuye a la introducción de novedades. El triunfo de la implantación requiere además un liderazgo sólido y una estrategia de introducción, viniendo acompañado frecuentemente del soporte de expertos externos. De otro modo, la implantación puede fracasar pues aparecen las mismas barreras que en las empresas de sectores manufactureros, además de la variabilidad propia de la explotación minera.

Las empresas del sector minero que han implantado algún tipo de herramienta de LM lo han hecho de manera similar a muchas empresas industriales: adoptando sólo ciertas herramientas y empezando por el área donde se ha creído más adecuado. Entre todas las experiencias prácticas recogidas en la literatura, se pueden hallar ejemplos de la gran mayoría de herramientas lean que emplean las empresas industriales por lo que parece que la aplicabilidad de dichas herramientas al sector minero puede darse por confirmada. De todos modos, la adopción de LM en el sector minero no es generalizada por lo que la divulgación de las mejores prácticas y de los resultados alcanzados va a tener un papel fundamental. Para los académicos, se abre una vía de investigación sobre las adaptaciones de herramientas concretas sobre la que actualmente se ha escrito muy poco. Para los profesionales del sector, la gestión lean y sus herramientas se presentan como un instrumento poderoso para relanzar la competitividad del sector en un momento clave. 


\section{Referencias}

ANÓNIMO (1989). "Knife River hones an edge". Coal Age, $26(9)$, pp. 63-64.

AZZAMOURI, A., FENIES, P., y FONTANE, F. (2016). "Une approche pour un Lean Mining Responsable: mise en oeuvre sur la chaîne logistique de l'OCP". En: 11es Rencontres Internationales de la Recherche en Logistique et Management de la Chaîne logistique. Lausanne 7 - 9 septembre 2016

BALADRÓN, C., y ALARCÓN, L.F. (2017). "Assessing the impact of lean methods in mining development projects". En: IGLC 2017 - Proceedings of the 25th Annual Conference of the International Group for Lean Construction, pp. 137-144.

BOAR, A., BASTIDA, R., y MARIMON, F. (2020). "A Systematic Literature Review. Relationships between the Sharing Economy, Sustainability and Sustainable Development Goals". Sustainability, 12, no. 6744.

Boateng-Okrah, E., y Appiah Fening, F. (2012). TQM implementation: a case of a mining company in Ghana". Benchmarking: An International Journal, 19 (6), pp. 743-759.

BRANDAO DE SOUZA, L. (2009) "Trends and approaches in lean healthcare". Leadership in Health Services, 22 (2), pp. 121-139.

BRODNY, J., y TUTAK, M. (2017). "Application of Elements of TPM Strategy for Operation Analysis of Mining Machine". En: IOP Conference Series: Earth and Environmental Science, 95 (4), art. no. 042019

CASTILlO, G., ALARCÓN, L.F., y GONZÁLEZ, V.A. (2014). "Evaluating the impact of lean methodologies in copper mining development projects". En: 22nd Annual Conference of the International Group for Lean Construction: Understanding and Improving Project Based Production, IGLC 2014, pp. 593-604.

CASTILlO, G., ALARCÓN, L.F., y GONZÁLEZ, V.A. (2015). "Implementing lean production in copper mining development projects: Case study". Journal of Construction Engineering and Management-ASCE, 141 (1), pp.05014013-1-11.

CAVENDER, B.W. (1993). "Application of 'world-class manufacturing' techniques to production milling operations". Mining Engineering, 45 (9), pp.:1152-1157.

CHADWICK, J. (1995). "Mine automation". Mining Magazine, 172 (6), pp. 333-343.
CHLEBUS, E., HELMAN, J., OLEJARCZYK, M., y ROSIENKIEWICZ, M. (2015). "A new approach on implementing TPM in a mine - A case study". Archives of Civil and Mechanical Engineering, 15 (4), pp. 873884.

CLAASSEN, J.O. (2016). "Application of manufacturing management and improvement methodologies in the southern African mining industry". Journal of the Southern African Institute of Mining and Metallurgy, 116 (2), pp. 139-148.

DIESTE, M., PANIZZOLO, R., GARZA-REYES, J.A., y ANOSIKE, A (2019) "The relationship between lean and environmental performance: Practices and measures". Journal of Cleaner Production, 224, pp. 120-131.

DUNSTAN, K., LAVIN B., y SANFORD, R. (2006). "The application of lean manufacturing in a mining environment". En: Australasian Institute of Mining and Metallurgy Publication Series, pp. 145-157.

EL PERIÓDICO. (2020). Descubiertos en Ourense minerales necesarios para el coltán. Disponible online en: https://www.elperiodico.com/es/economia/20200205/ obtienen-primera-vez-espana-el-oro-negro-7836223 Publicado 5 febrero de 2020. Acceso 1 diciembre 2020.

EUROPA (2009). "Dictamen del Comité Económico y Social Europeo sobre "La minería no energética" en Europa". Diario Oficial de la Unión Europea, C 27, 3 de febrero de 2009, pp. 82-97.

FLYNN, J.R., y VLOK, P.J. (2015). "Lean approaches in asset management within the mining industry". En: Amadi-Echendu J., Hoohlo C., Mathew J. (eds) 9th WCEAM Research Papers. Lecture Notes in Mechanical Engineering, 20, Cham: Springer, pp. 101-118.

FORTUNY-SANTOS, J. RUIZ-DE-ARBULO LÓPEZ, P. LUJÁN-BLANCO, I., y CHEN, P.K. (2020). "Assessing the synergies between lean manufacturing and Industry 4.0". Dirección y Organización, 71, pp. 71-86.

GROENEVELD, O.L. (2006). "The mine manager's toolkit". En: International Mine Management Conference : 16 - 18 October 2006, Melbourne, Victoria, Australia. Carlton, Vic. : Australasian Institute of Mining and Metallurgy, pp. 3-5.

HARAGOVICS, M., y MIZSEY, P. (2014). "A novel application of exergy analysis: Lean manufacturing tool to improve energy efficiency and flexibility of hydrocarbon processing". Energy, 77, pp. 382-390. 
HATTINGH. T., y KEYS, O. (2010). "How applicable is industrial engineering in mining?" En: 4th International Platinum Conference. Platinum in Transition 'Boom or Bust', Sun City, South Africa, pp. 205-210.

HAUGEN, S. (2013). "Lean mining". Mineralproduksjon, 3, B21-B40.

HELMAN, J. (2012). "Analysis of the potentials of adapting elements of Lean Methodology to the unstable conditions in the mining industry". AGH Journal of Mining and Geoengineering, 36 (3), pp. 151-157.

INDRAWATI, S. y RIDWANSYAH, M. (2015). "Manufacturing Continuous Improvement Using Lean Six Sigma: An Iron Ores Industry Case Application". Procedia Manufacturing, 4, pp. 528-534.

IRANMANESH, M., ZAILANI, S., HYUN, S.S., ALI, M.H., y KIM, K.Y. (2019). "Impact of Lean Manufacturing Practices on Firms' Sustainable Performance: Lean Culture as a Moderator". Sustainability, 11(4), no. 1112.

KĘSEK, M., BOGACZ, P., y MIGZA, M. (2019) "The application of Lean Management and Six Sigma tools in global mining enterprises". IOP Conference Series: Earth and Environmental Science, 214 (1), no. 012090.

KLIPPEL, A.F., PETTER, C.O, y ANTUNES Jr., J.A.V. (2008a). "Lean management implementation in mining industries". DYNA (Colombia), 75 (154), pp. 81-89.

KLIPPEL, A.F., PETTER, C.O, y ANTUNES Jr., J.A.V. (2008b). "Management Innovation, a way for mining companies to survive in a globalized world". Utilities Policy, 16 (4), pp. 332-333.

LIN, J., y GHODRATI, B. (2011) "A step-by-step model to improve delivery assurance: A case study in mining industry". En: 2011 IEEE International Conference on Quality and Reliability Quality and Reliability (ICQR), pp. 36-40.

LIU, Z.X. (2013). "Study on coal lean mining theory and practice". Advanced Materials Research, 605-607, pp. 538-541.

LƠ W, J. (2019). "An investigation into lean production practice in mining". International Journal of Lean Six Sigma, 10 (1), pp. 123-142.

MAKUMBE. S., HATTINGH, T., PLINT, N., y ESTERHUIZEN, D. (2018). "Effectiveness of using Learning Factories to impart Lean principles in mining employees". Procedia Manufacturing, 23, pp. 69-74.
MARTÍNEZ RAMOS, M. (2004). "Proceso, antecedentes y consecuencias de la adopción de innovaciones contables: contrastación de un modelo integrador". Cuadernos de Economía y Dirección de Empresas, 21, pp. 5-26.

MISHRA, D.P., SUGLA, M., y SINGHA, P. (2013). "Productivity Improvement in Underground Coal Mines - A Case Study". Journal of Sustainable Mining, 12 (3), pp. 48-53.

MOTTOLA, L. (2018). Why the mining industry desperately needs lean thinking. Disponible online en: https://planetlean.com/lean-management-mining-industry/ Acceso 1 diciembre de 2020 .

MIKHALCHENKO, V., y RUBANIK, Y. (2019). "Application of the "lean thinking" concept to the analysis of coal mining region's sustainable development objective" E3S Web of Conferences, 134, no. 03001.

MUSINGWINI, C., MINNITLT, R.C.A., PHUTI, D., y MAKWARA, F. (2003). "Just-in-time development model for a sub-level caving underground mine in Zimbabwe". Journal of The South African Institute of Mining and Metallurgy, 103 (3), pp. 187-191.

NADEAU, S., MORENCY, F., y NSANGOU, J.R. (2015). "The contextualisation of lean manufacturing in the mining sector: foreseeable challenges to occupational health and safety". En: Proceedings 19th Triennial Congress of the International Ergonomics Association (IEA), 9-14 August, 2015 (Melbourne, Australia).

NETLAND, T.H., y POWELL, D.J (eds.) (2017). The Rouledge companion to lean management, New York: Rouledge, Taylor \& Francis group.

OHNO, T. (1988). The Toyota Production System: Beyond Large-Scale Production, Portland, Oregon: Productivity Press.

PETTERSEN, J. (2009) "Defining lean production: some conceptual and practical issues". The TQM Journal, 21 (2), pp. 127-142.

ROBERTSON, J. (2019). Cobalto: qué pasó con la burbuja del "oro azul" (y cuándo volverá a reactivarse). Disponible online en: https://www.bbc.com/mundo/ noticias-internacional-49319245 Publicado 12 agosto de 2019. Acceso 1 de diciembre de 2020.

ROELANDT, K.F. (2008). "Implementing LEAN Construction in a South African Construction company". En: Proceedings of IGLC16: 16th Annual Conference of the International Group for Lean Construction, pp. 259267. 
RUIZ DE ARBULO LÓPEZ, P., FORTUNY SANTOS, J, VINTRÓ SÁNCHEZ, C., y BASÁÑEZ LLANTADA, A. (2013). "Aplicación de time-driven activity-based costing en la producción de componentes de automóvil". Dyna ingeniería e industria, 88 (2), pp. 234-240.

SANDA, M.A., JOHANSSON, J., y JOHANSSON, B. (2011). "Miners' tacit knowledge: A unique resource for developing human-oriented lean mining culture in deep mines". En: International Conference on Industrial Engineering and Engineering Management, no. 6117947 , pp. 399-404.

SAYERS, A. (2007). "Tips and tricks in performing a systematic review". British Journal of General Practice, 57 (538), p. 425.

SEIFULLINA, A. ER A, NADEEM, S.P., GARZA-REYES, J.A., y KUMAR, V. (2018). "A Lean Implementation Framework for the Mining Industry". IFAC PapersOnLine, 51 (11), pp. 1149-1154.

SHAH, R., y WARD, P. (2007). "Defining and developing measures of lean production". Journal of Operations Management, 25 (4), pp. 785-805.

SIMMONS, M.R. (1996), "Gulf of Mexico - 'silicon valley' to offshore oil \& gas industry". Sea Technology, 37 (8), pp. $27,28,30-32$.

SPECTOR, R. (2006). "How constraints management enhances lean and six sigma". Supply Chain Management Review, 10 (1), pp. 42-47.

STECUŁA, K., TUTAK, M., y BRODNY, J. (2017). "Application of chosen elements from Japanese production and maintenance management philosophies in Polish coal mines". En: International Multidisciplinary Scientific GeoConference Surveying Geology and Mining Ecology Management, SGEM, 17 (13), pp. 93100 .

STEINBERG, J.G., y DE TOMI, G. (2010). "Lean mining: Principles for modelling and improving processes of mineral value chains". International Journal of Logistics Systems and Management, 6 (3), pp. 279-298.

STRAUSS, S.D. (1989). "An inventory policy that went astray". Minerals and Energy - Raw Materials Report, 6 (4), pp. 32-35.

SUGIMORI, Y., KUSSUNOKI, K., CHO, F., y UCHIKAWA, S. (1977). "Toyota production system materialization of just-in-time and respect-for-human system". International Journal of Production Research, 15 (6), pp. $553-564$
TAVARES THOMÉ, A.M., SCAVARDA L.F., y SCAVARDA, A.J. (2016). "Conducting systematic literature review in operations management". Production Planning \& Control, 27 (5), pp. 408-420.

THUNNISSEN, M. y GALLARDO-GALLARDO, E. (2019). "Rigor and relevance in empirical TM research: Key issues and challenges". BRQ Business Research Quarterly, 22, pp. 171-180.

VALENT, M.C., HAYWOOD, G. (2002). "Yilgarn Star Just in Time Methodology - Planning and Scheduling Implications". In: Australasian Institute of Mining and Metallurgy Publication Series, 2002 (5), pp. 39-46.

VANEGAS, N. (1999). "Applications of discrete-event simulation in Canadian mining operations in the nineties". International Journal of Surface Mining, Reclamation and Environment, 13 (2), pp. 77-78.

VANEK, M., ŠPAKOVSKÁ, K., MIKOLÁŠ, M., y POMOTHY, L. (2015). "Continuous improvement management for mining companies". Journal of the Southern African Institute of Mining and Metallurgy, 115 (2), pp. 119-124.

WADE, J. (2008) "BHP moves into steel". Australian Mining, 100 (1), p. 12.

WIJAYA, A., KUMAR, R., y KUMAR, U. (2009). "Implementing lean principle into mining industry issues and challenges." En: International Symposium on Mine Planning and Equipment Selection, Banff, Canada, pp. 1-9.

WOMACK, J.P., JONES, D.T., y ROOS, D. (1990). La máquina que cambió el mundo, Barcelona: Profit (edición en español de 2017).

WONG, M. (2007). "The Role of Culture in Implementing Lean Production System". En: Olhager, J. y Persson, F (eds.) IFIP Intemational Federation for Information Processing. Advances in Production Management Systems, 246, Boston: Springer, pp. 413-422.

YINGLING, J.C., DETTY, R.B., y SOTTILE Jr., J. (2000). "Lean manufacturing principles and their applicability to the mining industry". Mineral Resources Engineering, 9 (2), pp. 215-238.

YU, H., SUN, X., y Solvang, W.D. (2016) "Implementation of Lean Philosophy and Lean Tools in Coal Mining Industry" In: Petrova, V.M. (ed) Advances in Engineering Research, 12, Nova Science Publishers, pp. 1-46.

YUAN, Q., YIN, X., JIA, S., y LI, Z. (2016) "A new approach of controlling medium consumption with applications in coal mining industry". International Journal of Performability Engineering, 12 (2), pp. 173-182. 